\title{
Point Shear Wave Elastography and the Effect of Physical Exercise, Alcohol Consumption, and Respiration in Healthy Adults
}

\section{(1) $(\Theta$}

Authors

Victoria Taraldsen', Sunneva Tomasgard1', Margrethe Thune Rudlang1, Odd Helge Gilja1, 2, Mette Vesterhus ${ }^{1,3,4}$, Anders Batman Mjelle ${ }^{1,5}$

Affiliations

1 Department of Clinical Science, University of Bergen Faculty of Medicine and Dentistry, Bergen, Norway

2 Haukeland University Hospital, National Centre for Ultrasound in Gastroenterology, Bergen, Norway

3 Department of Medicine, Haraldsplass Deaconess Hospital Bergen, Norway

4 Norwegian PSC Research Center (NoPSC), Division of Surgery, Inflammatory medicine and transplantation, Oslo Universitetssykehus Rikshospitalet, Oslo, Norway

5 Department of Clinical Medicine, Haukeland University Hospital, National Centre for Ultrasound in Gastroenterology, Bergen, Norway

Key words

Shear wave elastography, Physical exertion, Alcohol consumption, Respiration, ultrasound, Methods and techniques

received 28.04.2020

revised $\quad 17.08 .2020$

accepted 26.10.2020

published online 2020

Bibliography

Ultrasound Int Open 2020; 6: E54-E61

DOI 10.1055/a-1298-9642

ISSN 2199-7152

(c) 2020. The Author(s).

This is an open access article published by Thieme under the terms of the Creative Commons Attribution-NonDerivative-NonCommercial-License, permitting copying and reproduction so long as the original work is given appropriate credit. Contents may not be used for commecial purposes, or adapted, remixed, transformed or built upon. (https://creativecommons.org/ licenses/by-nc-nd/4.0/)

Georg Thieme Verlag KG, Rüdigerstraße 14,

70469 Stuttgart, Germany
Correspondence

Dr. Anders Batman Mjelle

Haukeland Universitetssjukehus, Pediatric and Adolescent

Medicine

Jonas Lies vei 65

5021 Bergen

Norway

Tel.: + 4755975000, Fax: +4755975147

abmjelle@gmail.com

\section{ABSTRACT}

Purpose Ultrasound elastography is a noninvasive method for liver stiffness measurement (LSM) with the aim of reflecting approximate liver fibrosis load. Despite minimal evidence, current guidelines recommend $10 \mathrm{~min}$ of rest and breath hold prior to measurements and offer no advice concerning recent alcohol consumption, leading to challenges in clinical practice. We aimed to investigate how LSM in healthy adults is influenced by physical exercise, recent alcohol consumption, and respiration.

Materials and Methods 42 healthy subjects aged 21-36 years were included. LSM using point shear wave elastography (pSWE) was performed in five stages: baseline, after physical activity, after registration of alcohol consumption, and during breath hold compared to free breathing.

Results LSM values were significantly increased following physical exercise compared to baseline values $(4.1 \pm 0.8$ vs. $3.8 \pm 0.8 \mathrm{kPa}, \mathrm{p}=0.01)$. Alcohol consumption during the last $72 \mathrm{~h}$ (0-27 alcohol units) did not significantly affect LSM. There was no significant difference between LSM during breath hold and free breathing.

Conclusion In healthy subjects, LSM increased after recent physical exercise, while alcohol consumption $24-72 \mathrm{~h}$ prior to examination did not have a significant impact. There was no clinically significant effect of breath hold on LSM. Our study supports present guidelines recommending rest prior to LSM, while indicating that breath hold may not be mandatory. Recent moderate alcohol exposure may affect LSM to a lesser extent than commonly believed. 


\section{Introduction}

Liver elastography is established as an important noninvasive tool for the evaluation of chronic liver diseases, allowing bedside assessment of liver elasticity as an estimation of fibrosis [1,2] and offering an assessment of disease stage, progression, and prognosis. Liver stiffness measurement by ultrasound elastography directly reflects a physical property of the liver, in contrast to serological tests of fibrosis which are influenced by fibrosis in other organs. However, various exposures (e. g. deep inspiration, recent meal intake) and underlying conditions (e.g. right heart failure) other than liver fibrosis have been demonstrated to affect liver stiffness measurements (LSM) [3-5].

In order to enable the comparison of LSM to established cut-off values, between centers, and in repeated measurements over time in the follow-up of individual patients, current international guidelines provide recommendations regarding the standardization of the performance of liver elastography examinations and common quality criteria for their interpretation. A minimum of 10 min rest prior to examination and a mid-respiratory breath hold are explicitly recommended [2]. Furthermore, guidelines state that LSM decreases following 1-4 weeks of detoxification in subjects with alcoholic hepatitis, and that there is insufficient data to evaluate the use of shear wave elastography (SWE) in populations with chronic alcohol overuse [2]. Data concerning recent alcohol exposure prior to LSM in healthy adults without chronic alcohol overuse or alcoholic liver disease are scarce $[2,6]$, and recommendations in international guidelines are lacking, giving rise to a lack of certainty among clinicians and patients.

Breath hold has been identified as time-consuming and as an important limiting factor for clinical implementation of liver elastography, as not all patients are able to hold their breath [7]. Furthermore, the overall cost of cancelled appointments or invalid examinations due to individuals not fulfilling the demanding and con- ceivably unnecessary instructions might be reduced if evidence is provided to simplify preparations prior to LSMs [2]. Considering the benefits of time and cost effectiveness, we aimed to investigate the impact of recent physical exercise, recent alcohol consumption, and calm free breathing during LSM.

\section{Materials and Methods}

\section{Subjects}

This prospective cross-sectional study was performed at a single university hospital between April and June 2019. 42 healthy volunteers ( 21 males, $50 \%$ ) were enrolled. The mean \pm SD age was $25.7 \pm 3.1$ years. Structured patient interviews and a standardized abdominal B-mode ultrasonography examination were performed in all subjects prior to LSM. The exclusion criteria comprised a previous history of disease in the liver, heart, or respiratory system, previous or ongoing malignancy, inflammatory bowel disease, pregnancy, any liver pathology on B-mode ultrasound, or a $\mathrm{BMI} \geq 30 \mathrm{~kg} / \mathrm{m}^{2}$. After excluding 1 subject, 41 (97.6\%) subjects were included. Weight and height were measured to calculate BMI. Some of the subjects participated in parts of the study ( $\vee$ Fig. 1).

\section{Investigation procedure}

Liver stiffness measurement (LSM) by point shear wave elastography (pSWE) was performed using Samsung RS80A with Prestige (Samsung Medison Co, Ltd, Seoul, Korea) with a CA1-7A convex array probe (1-7 MHz). Measurements were obtained in the right liver lobe with participants lying in a supine position with the right arm maximally abducted during a short mid-respiratory breath hold. The transducer was placed perpendicularly in an intercostal space, while applying minimal pressure. The region of interest (ROI) had a fixed height of $10 \mathrm{~mm}$ and was placed in a homogeneous area

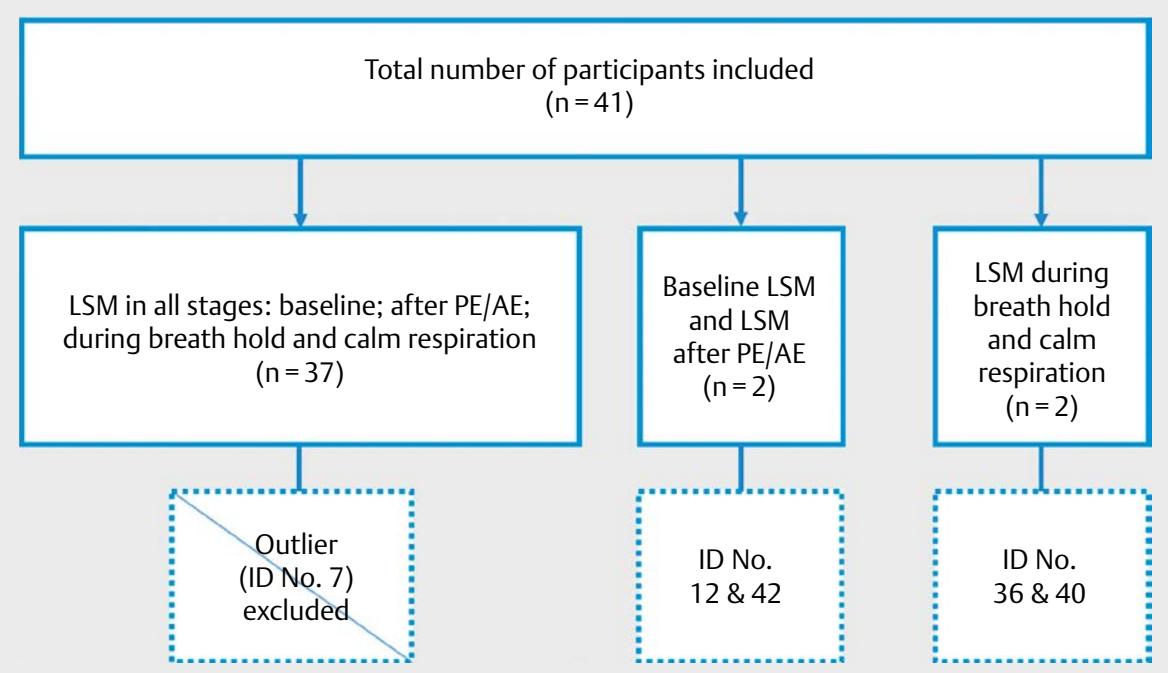

-Fig. 1 Flowchart for study participants. The flowchart shows inclusion of participants. All underwent liver stiffness measurements (LSM), either in all stages including baseline, physical exercise (PE), alcohol exposure (AE) and different respiratory phases; in baseline and after PE/AE; or only LSM in different respiratory phases. 
2-5 centimeters beneath the liver capsule, avoiding visible biliary tracts and vessels. Values were expressed in kilopascals ( $\mathrm{kPa})$. To obtain a valid measurement, 10 acquisitions were required, with an interquartile range/median ratio $(I Q R / M) \leq 30 \%$. If the $I Q R / M$ exceeded $30 \%$, data were erased, and the examination was repeated once with 10 novel acquisitions and kept if the IQR/M was $\leq 30 \%$ $(n=2)$.

LSM was performed by three operators, all medical students (MR, VT, ST) who received training from experienced liver elastography users (ABM, MV) prior to the start of the study. The training period finished when LSM was adequately performed in a standardized fashion. Supervision was maintained throughout the study period to ensure correct technique. Two operators performed baseline examinations as well as LSM after exercise or alcohol together: one (MR or VT) holding the probe, one controlling the ultrasound scanner (MR or VT). For LSM evaluating the effect of breath hold versus free breathing, LSM was performed by a third operator (ST).

Participants were instructed not to consume alcohol 5 days prior to baseline measurements, and fasted $\geq 3 \mathrm{~h}$ prior to every measurement, including caffeine and chewing gum. Water consumption was restricted to one glass of $50 \mathrm{ml}$. Prior to measurements, subjects rested in a chair for 15 min prior to measurements, except when tested for the effect of physical exercise. Measurements after exercise and consumption of alcohol were conducted on two separate days, following the same procedure.

On the first test day, baseline LSM was performed in all subjects. Participants were then instructed to run up and down five flights of stairs. The pulse was manually recorded immediately before and after the run. LSM was repeated when the candidate was able to perform the necessary breath hold. On the second test day, chosen to be a Monday as alcohol consumption is known to be higher during weekends, participants registered the number of alcohol units consumed during the last $72 \mathrm{~h}$. One unit of alcohol was defined according to national guidelines: 12 grams of pure alcohol, corresponding to a small glass $(125 \mathrm{ml})$ of wine, a shot $(40 \mathrm{ml})$ of strong liquor (35-40\%) or 0.33 liters of beer. LSM was conducted as previously described. LSM was then performed twice more in the same participants: the first LSM during calm free breathing, the second with mid-expiratory breath hold.

\section{Ethical aspects}

The protocol was in accordance with the Declaration of Helsinki and approved by The Regional Committee on Medical and Health Research Ethics. Written informed consent was obtained from all subjects following both oral and written information.

\section{Statistics}

All statistical analyses were performed using IBM SPSS Statistics 25 Software (SPSS Inc., 2016 Armonk, NY). The data were assessed using normality tests of Shapiro-Wilk. Histograms and Q-Q plots were produced to visualize the distribution of the data. For all normally distributed variables, the mean \pm standard deviation (SD) was calculated, and for variables not normally distributed, the median [range] was calculated. For comparison between groups or repeated measures, parametric (e.g. paired t-tests) or nonparametric tests (Wilcoxon signed-rank test) were applied when appropriate.
Correlations and unequal distributions were tested by the Pearson correlation and Bland-Altman plots. Statistical significance was defined as a $\mathrm{p}$-value $<0.05$.

\section{Results}

A total of 41 healthy subjects were included in the final analysis (20 men [51.3\%]) following exclusion of one female extreme outlier with consistently high baseline LSM values at repeated measurements: although thorough investigations including viral, hematological, and immunological screening, second opinion LSM on a different elastography platform, and ultrasonography of the abdomen did not reveal any other signs of liver disease, LSM values were normalized at control several weeks later and an intercurrent liver affection could not be ruled out. LSM for the entire cohort was $3.8 \pm 0.8 \mathrm{kPa}$. There was no difference in baseline LSM between females and males ( $3.8 \pm 0.7$ vs. $3.7 \pm 0.9 \mathrm{kPa}, \mathrm{p}=0.76)$.

Valid LSM values were obtained in all included subjects $(n=39)$ for every different measurement type: at baseline, following physical activity and alcohol exposure, and during breath hold and free breathing ( $\triangleright$ Fig. 2 ; $\triangleright$ Table 1 ). Baseline characteristics for participants are displayed in $>$ Table 2.

\section{Effects of physical exercise prior to LSM}

Compared to baseline, the mean LSM value after physical strain was significantly higher for the entire population $4.1 \pm 0.8 \mathrm{vs} .3 .8 \pm 0.8 \mathrm{kPa}$ $(p=0.01)$. This effect seemed to be gender-specific, as the significant increase only concerned males $(4.2 \pm 0.5 \mathrm{kPa}$ vs. $3.8 \pm 0.7 \mathrm{kPa}$, $p=0.02)$, and not females $4.0 \pm 1.1$ vs. $3.7 \pm 0.9, p=0.18)$. Clinically significant differences ( $\geq 1 \mathrm{kPa}$ ) were found in $8 / 39$ (20.5\%), the maximum difference was $+2.7 \mathrm{kPa}$. Seven of these were positive, including three females.

Participants $(n=9)$ with an exercise-induced increase less than 40 beats per minute (bpm) did not show a change in LSM $(4.0 \pm 0.5$ vs. $3.9 \pm 0.8 \mathrm{kPa}, \mathrm{p}>0.7)$, while LSM increased in subjects with a pulse increase $\geq 40 \mathrm{bpm}(4.2 \pm 0.9$ vs. $3.7 \pm 0.7 \mathrm{kPa}, \mathrm{p}=0.01)$. Pulse change was equal across genders.

\section{Effect of alcohol $72 \mathrm{~h}$ prior to LSM}

36 participants consumed alcohol during the registration period, reporting a median [range] amount of 7.5 [1.5-27] units, while 3 subjects remained abstinent. The historical median weekly alcohol consumption reported among alcohol consumers, was 5.6 units, possibly suggesting that the alcohol consumption during the three days prior to test day 2 was higher than normal, but the difference was not significant $(p=0.13)$. Alcohol was consumed by 14,14 , and 23 participants on day one, day two, and day three before LSM, respectively. The corresponding number of subjects consuming $\geq 5$ units of alcohol on a single day was 4, 8 , and 15 , respectively.

LSM on test day 2 (following alcohol registration) was not significantly different from baseline among participants who consumed alcohol during the last $72 \mathrm{~h}(\mathrm{n}=36)(3.7 \pm 0.7 \mathrm{vs} .3 .8 \pm 0.8 \mathrm{kPa}$, $\mathrm{p}=0.58)$. Dot plot did not indicate any tendency for correlation of LSM with recent alcohol consumption ( $>$ Fig. 3 ). Clinically significant differences ( $\geq 1 \mathrm{kPa}$ ) were found in $11 / 39(28.2 \%)$, with a maximum difference of $-2.9 \mathrm{kPa}$. 


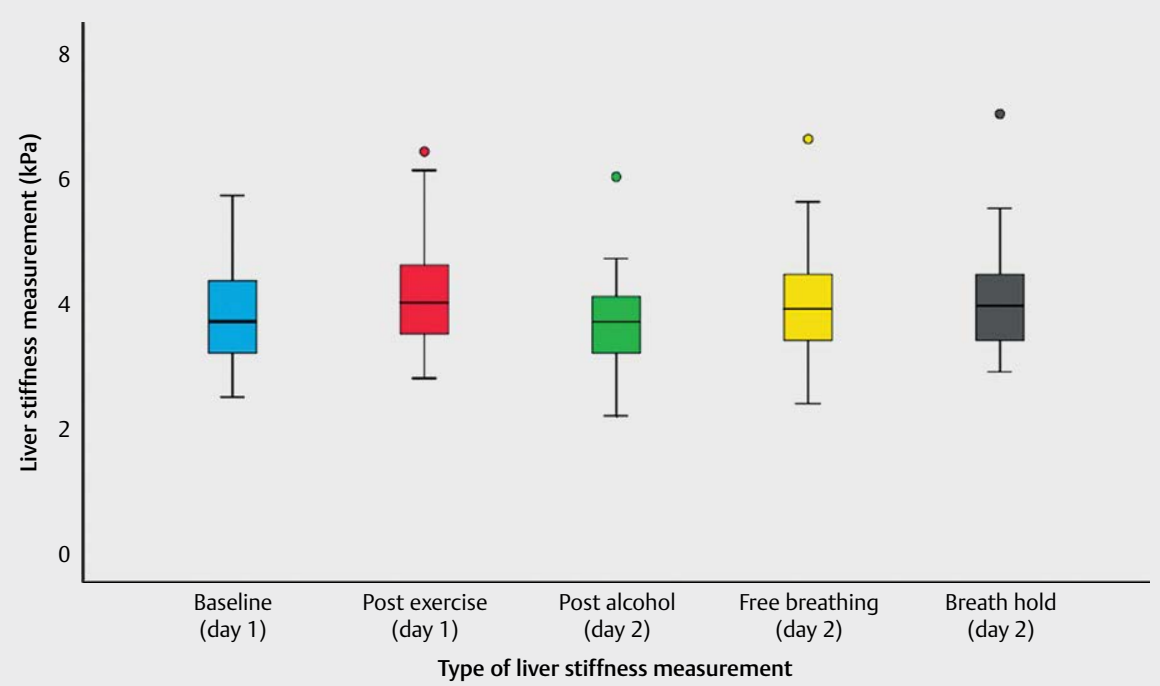

- Fig. 2 Liver stiffness measurements based on LSM measurement category. Baseline measurements and after physical exercise (day 1 ) and after registration of alcohol exposure and during different respiratory phases (day 2). Significant difference only between baseline LSM and LSM post-exercise $(p=0.01)$.

- Table 1 Liver stiffness measurements for the entire panel $(n=41)$ and across genders, in all 5 stages: baseline LSM; LSM after exercise; LSM after alcohol consumption registration; LSM during breath hold; LSM during calm respiration.

\begin{tabular}{|l|l|l|l|l|}
\hline & $\begin{array}{l}\text { Total } \\
\text { panel }\end{array}$ & Males & Females \\
\hline $\begin{array}{l}\text { Liver stiffness } \\
\text { measurement }\end{array}$ & Day 1: Baseline & $3.8 \pm 0.8$ & $3.8 \pm 0.7$ & $3.7 \pm 0.9$ \\
\hline $\begin{array}{l}\text { (LSM), kPa, } \\
\text { mean } \pm \text { SD }\end{array}$ & $\begin{array}{l}\text { Day 1: } \\
\text { Post-exercise }\end{array}$ & $4.1 \pm 0.8$ & $4.2 \pm 0.5$ & $4.0 \pm 1.1$ \\
\hline & $\begin{array}{l}\text { Day 2: } \\
\text { Post-alcohol }\end{array}$ & $3.7 \pm 0.7$ & $3.8 \pm 0.7$ & $3.5 \pm 0.7$ \\
\hline & $\begin{array}{l}\text { Day 2: Breath } \\
\text { hold }\end{array}$ & $4.0 \pm 0.8$ & $3.9 \pm 0.9$ & $4.1 \pm 0.7$ \\
\hline & $\begin{array}{l}\text { Day 2: Calm } \\
\text { respiration }\end{array}$ & $3.9 \pm 0.8$ & $3.9 \pm 0.8$ & $4.0 \pm 0.8$ \\
\hline
\end{tabular}

Participants $(n=11)$ reporting potential harmful alcohol use, defined as $\geq 14$ units/week for men and $\geq 9$ units/week for women, had similar baseline LSM values compared to other participants (3.8 \pm 0.7 vs. $3.7 \pm 0.8, p=0.82)$ and showed no alterations following alcohol consumption when compared to baseline $(3.8 \pm 0.5 \mathrm{vs}$. $3.8 \pm 0.7 \mathrm{kPa}, \mathrm{p}=0.91)$.

\section{LSM during calm respiration vs. breath hold}

There was no difference in mean LSM for the total panel between measurements during breath hold and calm respiration ( $4.0 \pm 0.8$ vs. $3.9 \pm 0.8 \mathrm{kPa}, \mathrm{p}=0.34)$. There was a tendency toward higher LSM during breath hold in females, but this was not significant ( $4.1 \pm 0.7$ vs. $4.0 \pm 0.8, p=0.06)$. The correlation between LSM in the two breathing states in the individual subject was good $(\mathrm{rho}=0.82$,
- Table 2 Background characteristics of all 41 participants. Data presented as mean \pm SD unless otherwise specified.

\begin{tabular}{|c|c|c|c|}
\hline & Total panel & Males & Females \\
\hline Age, years & $25.7 \pm 3.1$ & $26.3 \pm 3.6$ & $25.1 \pm 2.4$ \\
\hline $\begin{array}{l}\text { Body mass index (BMI), } \\
\mathrm{kg} / \mathrm{m}^{2}\end{array}$ & $23.8 \pm 3.0$ & $25.4 \pm 2.2$ & $22.1 \pm 2.9$ \\
\hline $\begin{array}{l}\text { Alcohol consumption last } \\
\text { month, units }\end{array}$ & $\begin{array}{l}26.6 \pm 23.3 \\
(0-120)\end{array}$ & $\begin{array}{l}23.7 \pm 18.8 \\
(2-80)\end{array}$ & $\begin{array}{l}29.6 \pm 27.4 \\
(0-120)\end{array}$ \\
\hline $\begin{array}{l}\text { Potentially harmful } \\
\text { monthly alcohol use, n (\%) }\end{array}$ & $12(30 \%)$ & $7(35 \%)$ & $4(21 \%)$ \\
\hline $\begin{array}{l}\text { Alcohol consumption in the } \\
\text { last } 72 \mathrm{~h} \text { prior to LSM day } 2\end{array}$ & $\begin{array}{l}8.7 \pm 7.0 \\
(0-27)\end{array}$ & $\begin{array}{l}10.1 \pm 7.0 \\
(0-24)\end{array}$ & $\begin{array}{l}7.2 \pm 6.8 \\
(0-27)\end{array}$ \\
\hline $\begin{array}{l}\text { Number of participants } \\
\text { (average number of units) } \\
\text { drinking alcohol }<24 \mathrm{~h} \\
\text { prior to LSM day } 2\end{array}$ & $14(4.3)$ & $7(3.6)$ & $7(4.9)$ \\
\hline $\begin{array}{l}\text { Number of participants } \\
\text { (average number of units) } \\
\text { drinking alcohol } 24-47 \mathrm{~h} \\
\text { prior to LSM day } 2\end{array}$ & $14(5.8)$ & $7(8.4)$ & $7(3.3)$ \\
\hline $\begin{array}{l}\text { Number of participants } \\
\text { (average number of units) } \\
\text { drinking alcohol } 48-72 \mathrm{~h} \\
\text { prior to LSM day } 2\end{array}$ & $23(8.6)$ & $12(9.8)$ & $11(7.3)$ \\
\hline
\end{tabular}

$p<0.001)$, with only $1 / 39$ (2.6\%) differences being clinically significant ( $\geq 1.0 \mathrm{kPa}$ ), the maximum difference noted was $1.0 \mathrm{kPa}$.

Analyzing the effect of breathing pattern on LSM quality criteria, we found an increased $\mathrm{IQR} / \mathrm{M}$ in men during calm respiration, as compared to measurements during breath hold $(17.8 \pm 5.3 \%$ vs. 


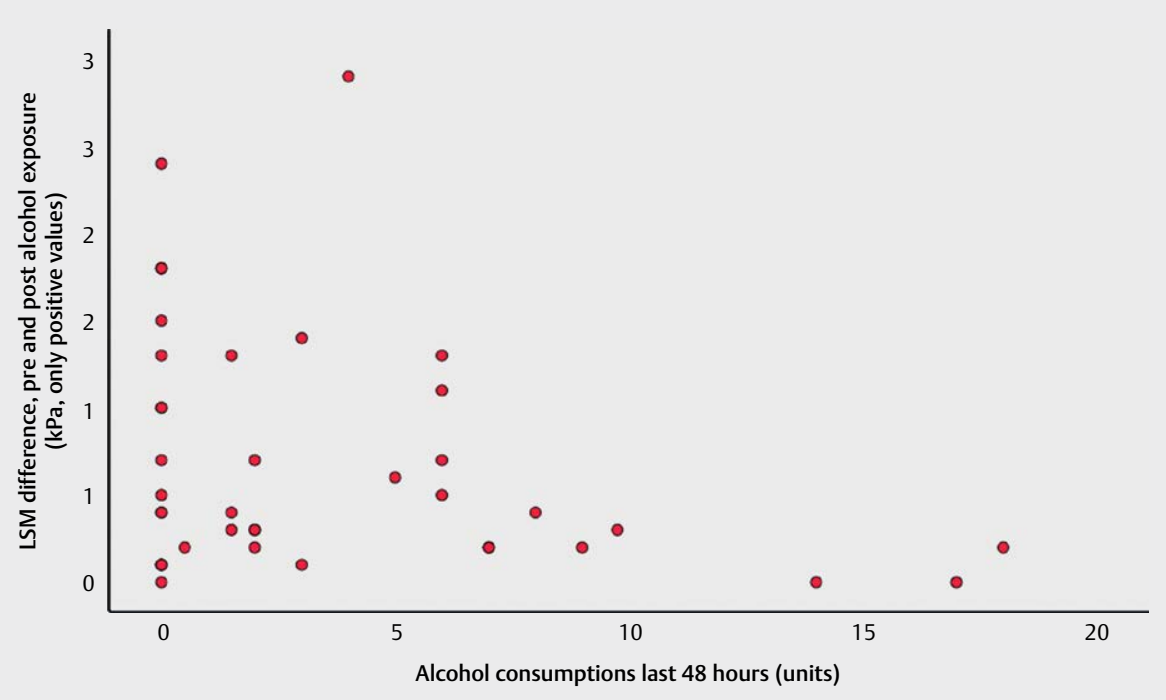

- Fig. 3 Scatter plot with alcohol consumption in units plotted against LSM difference from baseline, with all differences converted to positive values. No tendency of increasing LSM discrepancy after heavy alcohol intake, with almost no LSM change in individuals having consumed $\geq 7$ units the last $48 \mathrm{~h}$. The seeming tendency of less LSM difference with increasing alcohol consumption was not significant $(\mathrm{rho}=-0.27, p=0.1)$.

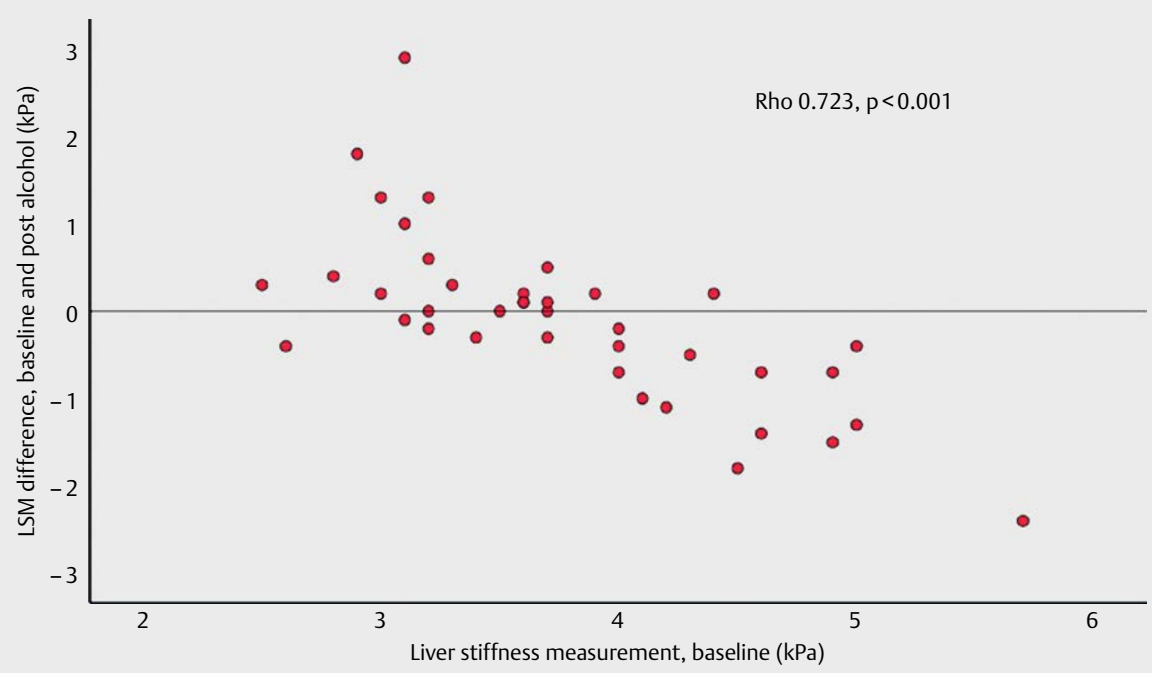

- Fig. 4 Scatter plot showing the correlation between baseline LSM and change in LSM on day 2 (after registration of alcohol consumption), demonstrating that individuals with a low baseline LSM often had an increase in LSM on day 2, while the opposite was true for individuals with a high baseline LSM.

$13.7 \pm 5.4 \% ; p=0.03)$, whereas no such difference was demonstrated in females or in the total cohort ( $p=0.68$ and 0.07 , respectively).

\section{Baseline LSM and LSM change}

Baseline LSM was the main determinant factor affecting change in LSM following exposure to exercise or various breathing patterns. When the baseline LSM was low, LSM often increased on subsequent measurements as illustrated in > Fig. 4, showing a strong correlation (rho 0.723, p<0.001), and > Fig. 5a-b, suggesting regression towards the mean as an explanatory factor.

\section{Discussion}

The introduction of liver elastography has improved the follow-up of patients with chronic liver disease, offering an easily accessible tool for real-time, noninvasive diagnosis and monitoring of liver fibrosis development. International guidelines, aiming to standardize examination procedures and quality criteria in an important effort to secure reliability and validity of results, are continuously being updated in response to the technological development in the field. However, for applicability as well as cost-effectiveness purposes, all recommendations with implications for practical appli- 

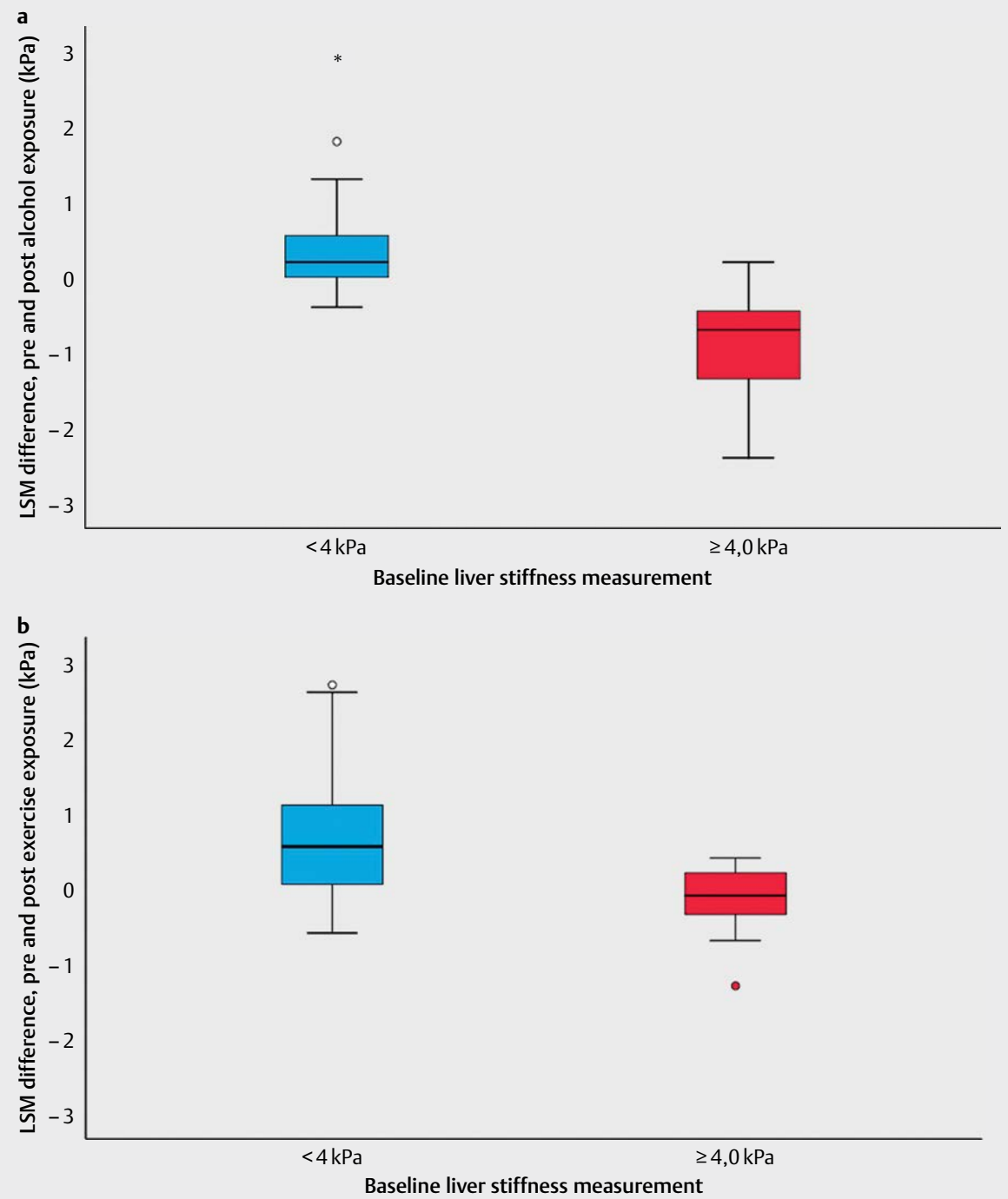

- Fig. 5 Boxplot with baseline liver stiffness measurements (LSM) divided into $\leq 4$ and $\geq 4 \mathrm{kPa}$, with change in LSM on the $y$-axis. a Difference between LSM after alcohol exposure and baseline LSM. $\mathbf{b}$ difference between LSM after physical activity and baseline LSM.

cation in clinical practice should be evidence-based, avoiding precautions complicating the procedure if not strictly necessary. Thus, it is important to thoroughly evaluate the clinically significant effect on LSM of the various procedure-related recommendations to identify the preparations truly required for performing valid liver elastography.

We found that LSM increased significantly following physical exercise, observing clinically significant differences ( $\geq 1 \mathrm{kPa}$ ) in 1 in 5 subjects.

Our study thus supports previous findings, strengthening the hypothesis that physical strain prior to LSM results in significantly higher LSM values and should be avoided prior to measurement. To our knowledge, only one other study has previously investigated the impact of exercise on LSM [8], but includes only 7 participants. The study found a significant LSM increase 0 and 5 min after activity, while it returned close to baseline values after $10 \mathrm{~min}$.
Pulse increase during physical strain has been linked to increased blood flow through the liver [9], further suggesting that blood redistribution may increase LSM [8]. In line with this, we observed that the increase in exercise-induced LSM was restricted to subjects with a pulse increase $>40 \mathrm{bpm}$, whereas individuals with a low pulse increase ( $\leq 40 \mathrm{bpm}$ ) did not show signs of increasing LSM after activity, suggesting that only strenuous exercise inducing a high heart rate affects LSM values. Hence, 10 min of rest prior to LSM may be superfluous in most subjects if strenuous exercise is avoided. The low number of individuals $(n=9)$ and short observation time following exercise preclude definitive conclusions, and further studies are warranted to strengthen recommendations regarding rest prior to LSM. Gender differences found in our study, i. e., significant LSM increase observed in males and not in females, also warrant further study to allow conclusions. 
To the best of our knowledge, this is the first prospective study examining the effects of recent alcohol consumption on LSM in healthy individuals. We found no significant effect on LSM following alcohol consumption 24-72 h prior to measurements, even in individuals with high recent consumption. Whether alcohol intake affects LSM in patients with chronic liver disease remains unknown and further investigations are needed.

Data on liver elastography and alcohol are limited and mostly retrospective $[6,10-14]$. There is insufficient evidence to evaluate the role of pSWE in alcoholic liver disease, as there are only three small studies on the use of pSWE for assessing alcoholic liver fibrosis, with inconsistent cut-off values for distinguishing absent or mild fibrosis from significant or severe fibrosis and cirrhosis $[2,12,13]$.

The lack of a generally accepted definition of potentially harmful weekly alcohol use is challenging in research regarding the effects of alcohol on the liver. Participants in our study meeting the criteria for potential harmful alcohol intake, applying Swedish guidelines citing a limit of 14 units per week for men and 9 units per week for women [15], did not show a higher baseline LSM or increasing LSM with recent alcohol exposure. Our findings may indicate that recent alcohol intake in healthy individuals does not affect LSM. However, since there were few subjects binge drinking the day prior to LSM $(n=4)$, we cannot exclude that a very high alcohol intake the night before LSM could cause transient liver inflammation with a corresponding LSM increase, or affect LSM through the induction of body water imbalance or dehydration. Our findings do not automatically transfer to chronic liver patients, and investigations in chronic liver patients are warranted.

In our study, we found no significant impact of breath hold on LSM values. Only a few studies have previously assessed the effect of respiration on LSM, and results are inconsistent regarding direct impact. Furthermore, studies have been inconsistent concerning methodology, systems used, and study population demography, precluding definitive conclusions regarding any impact of normal respiration [7, 16-19]. Some studies suggest that for certain elastography methods, deep inspiration and factors that decrease hepatic venous return (e.g. heart congestion, Valsalva maneuver), falsely increase LSM values, thereby overestimating cirrhotic changes $[5,20]$. It has been reported that different disease entities like hepatitis, alcoholic liver disease, and cirrhosis may respond differently regarding LSM changes when exposed to respiratory motion $[17,20]$.

Some studies question whether displacement of the liver may yield increased or inaccurate LSM values, or if the fast speed of the shear waves can somehow compensate for the motion effect $[2,16,17,21]$.

In a study investigating 123 adults with chronic liver disease using TE, free-breathing values in the expiration phase were higher compared to the inspiration phase, suggesting that liver decompression during free breathing may have a false-positive effect on LSM. However, free breathing was not compared to breath hold [17]. In contrast, two studies investigating 2D-SWE during calm free expiratory breathing versus breath hold in children, found no significant difference concerning respiratory motion and LSM changes [16, 22]. This corresponds well with our results using pSWE in healthy adults.
Breath hold is challenging for some patients and is not always applicable in clinical practice [2]. Previous studies adopting a free breathing pattern during examination $[7,23]$ have shown the free breathing approach to be beneficial regarding both applicability and time effectiveness. This resonates with our experience that measurements taken in free respiration are more time efficient and less demanding for the subjects, especially regarding apneal recovery.

We also describe an example of regression towards the mean, a statistical phenomenon arising when a random variable is extreme on the first observation but closer to the mean on the second observation ( $\vee$ Fig. 4,5 a-b) [24]. LSM acquisitions will often have a wide dispersion and vary even in controlled circumstances. In the case of a cohort of subject with a true LSM of $5.0 \mathrm{kPa}$, with LSM values varying between 4.5 and $5.5 \mathrm{kPa}$, subjects with a first LSM of $4.5 \mathrm{kPa}$ are likely to have a higher second LSM, while the opposite is true for those with a first LSM of $5.5 \mathrm{kPa}$. This must be taken into account when performing similar studies.

\section{Limitations}

The study design enabled the subjects to function as their own controls, allowing us to accept a smaller sample size, although a larger sample size might have been optimal for subanalyses per gender.

We considered the chance of unknown hepatic disease to be very low, as the study population consisted largely of young, nonobese, healthy students, with no history of serious illness and from an area (Norway) with a very low prevalence of viral hepatitis in the general population. Thus, the lack of relevant blood tests is a relatively minor limitation. Furthermore, all included participants showed LSM results within the normal range.

Alcohol intake was not standardized (for ethical reasons) and there is also a possibility of recollection bias regarding the subjects' recollection of the number of alcohol units consumed during the period of $72 \mathrm{~h}$ prior to LSM. Furthermore, fluid balance is a possible confounder after alcohol consumption, and measuring blood pressure and weight both before and after alcohol exposure would strengthen the study. This is, however, less likely given our negative findings.

The effect of breath hold was studied on day 2, after alcohol exposure. Although we made no observations pointing to difficulties during the study on day 2 , we cannot know for certain that this did not have an impact.

Our study population included only healthy young adults and cannot be directly extrapolated to chronic liver patients. The normal range is wide in our material as well as in the published literature, and it is likely that day-to-day and intra-individual variations of LSM affected our measurements.

\section{Conclusion}

We found that physical exercise led to increased LSM. However, this was restricted to individuals with a considerable heart rate increase following exercise, suggesting that current general recommendations for rest might be modified to advise patients to avoid strenuous exercise prior to liver elastography. Furthermore, our findings suggest that point shear wave elastography may be performed during calm respiration without inducing clinically significant affection of LSM in healthy adult livers. Further studies in chronic liver pa- 
tients are warranted to decide whether recommendations regarding breath hold should be modified. Alcohol exposure in the 24-72 $\mathrm{h}$ before examination did not affect LSM in healthy adults.

\section{Conflict of Interest}

OHG did consultancy for Samsung in 2017.

\section{References}

[1] Ferraioli G, Wong VWS, Castera L et al. Liver Ultrasound Elastography: An Update to the World Federation for Ultrasound in Medicine and Biology Guidelines and Recommendations. Ultrasound Med Biol 2018; 44: $2419-2440$

[2] Dietrich CF, Bamber J, Berzigotti A. EFSUMB Guidelines and Recommendations on the Clinical Use of Liver Ultrasound Elastography, Update 2017 (Long Version) (vol 38, pg e16, 2017). Ultraschall in Med 2017; 38: E52-E52

[3] Karlas T, Pfrepper C, Wiegand ] et al. Acoustic radiation force impulse imaging (ARFI) for non-invasive detection of liver fibrosis: Examination standards and evaluation of interlobe differences in healthy subjects and chronic liver disease. Scand J Gastroenterol 2011; 46: 1458-1467

[4] Arena U, Platon ML, Stasi C et al. Liver stiffness is influenced by a standardized meal in patients with chronic hepatitis c virus at different stages of fibrotic evolution. Hepatology 2013; 58: 65-72

[5] Lebray P, Varnous S, Charlotte F et al. Liver stiffness is an unreliable marker of liver fibrosis in patients with cardiac insufficiency. Hepatology 2008; 48: 2089-2089

[6] Bardou-Jacquet E, Legros L, Soro D et al. Effect of alcohol consumption on liver stiffness measured by transient elastography. World J Gastroenterol 2013; 19: 516-522

[7] Yoon HM, Cho YA, Kim JR et al. Real-time two-dimensional Shear-wave elastography for liver stiffness in children: Interobserver variation and effect of breathing technique. Eur J Radiol 2017; 97: 53-58

[8] Gersak MM, Sorantin E, Windhaber J et al. The influence of acute physical effort on liver stiffness estimation using Virtual Touch Quantification (VTQ). Preliminary results. Med Ultrason 2016; 18: 151-156

[9] Osada T, Iwane H, Katsumura T et al. Relationship between reduced lower abdominal blood flows and heart rate in recovery following cycling exercise. Acta Physiol 2012; 204: 344-353

[10] Tsochatzis EA, Gurusamy KS, Ntaoula S et al. Elastography for the diagnosis of severity of fibrosis in chronic liver disease: A meta-analysis of diagnostic accuracy. J Hepatol 2011; 54: 650-659

[11] Trabut JB, Thepot V, Nalpas B et al. Rapid decline of liver stiffness following alcohol withdrawal in heavy drinkers. Alcohol Clin Exp Res 2012; 36: 1407-1411

[12] Kiani A, Brun V, Laine F et al. Acoustic radiation force impulse imaging for assessing liver fibrosis in alcoholic liver disease. World J Gastroenterol 2016; 22: 4926-4935

[13] Lombardi R, Buzzetti E, Roccarina D et al. Non-invasive assessment of liver fibrosis in patients with alcoholic liver disease. World J Gastroenterol 2015; 21: 11044-11052

[14] Thiele M, Detlefsen S, Moller LS et al. Transient and 2-dimensional shear-wave elastography provide comparable assessment of alcoholic liver fibrosis and cirrhosis. Gastroenterology 2016; 150: 123-133

[15] Folkhälsomyndigheten (Public health agency of Sweden). https:// www.folkhalsomyndigheten.se/livsvillkor-levnadsvanor/alkohol-narkotika-dopning-tobak-och-spel-andts/alkohol/alkoholkonsumtion/. In
[16] Jung C, Groth M, Petersen KU et al. Hepatic shear wave elastography in children under free-breathing and breath-hold conditions. Eur Radiol 2017; 27: 5337-5343

[17] Yun MH, Seo YS, Kang HS et al. The effect of the respiratory cycle on liver stiffness values as measured by transient elastography. J Viral Hepat 2011; 18: 631-636

[18] Hong EK, Choi YH, Cheon JE et al. Accurate measurements of liver stiffness using shear wave elastography in children and young adults and the role of the stability index. Ultrasonography 2018; 37: 226-232

[19] Shin HJ, Kim MJ, Kim HY et al. Optimal acquisition number for hepatic shear wave velocity measurements in children. Plos One 2016; 11

[20] Horster S, Mandel P, Zachoval R et al. Comparing acoustic radiation force impulse imaging to transient elastography to assess liver stiffness in healthy volunteers with and without valsalva manoeuvre. Clin Hemorheol Microcirc 2010; 46: 159-168

[21] Bailey SS, Youssfi M, Patel M et al. Shear-wave ultrasound elastography of the liver in normal-weight and obese children. Acta Radiol 2017; 58: 1511-1518

[22] Franchi-Abella S, Corno L, Gonzales E et al. Feasibility and Diagnostic Accuracy of Supersonic Shear-Wave Elastography for the Assessment of Liver Stiffness and Liver Fibrosis in Children: A Pilot Study of 96 Patients. Radiology 2016; 278: 554-562

[23] Mjelle AB, Mulabecirovic A, Havre RF et al. Normal liver stiffness values in children: A comparison of three different elastography methods. J Pediatr Gastroenterol Nutr 2019; 68: 706-712

[24] Stigler S. Regression towards the mean, historically considered. Stat Methods Med Res 1997; 6: 103-114

\section{ERRATUM}

Victoria Taraldsen, Sunneva Tomasgard, Margrethe Thune Rudlang, Odd Helge Gilja, Mette Vesterhus, Anders Batman Mjelle.

Point Shear Wave Elastography and the Effect of Physical Exercise, Alcohol Consumption, and Respiration in Healthy Adults.

Ultrasound Int Open 2020; 06 (03): E54-E61

DOI: $10.1055 / a-1298-9642$

In the above article, names of three co-authors were indicated incorrectly.

Correct:

Margrethe Thune Rudlang, Odd Helge Gilja,

Anders Batman Mjelle

\section{Notice}

This article was changed according to the Erratum on Dec 23, 2020.

\section{Erratum}

In the above article, names of three co-authors were indicated incorrectly.

Correct:

Margrethe Thune Rudlang, Odd Helge Gilja, Anders Batman Mjelle 